\title{
Polypyrimidine tract-binding protein 1 regulates the Sertoli cell blood-testis barrier by promoting the expression of tight junction proteins
}

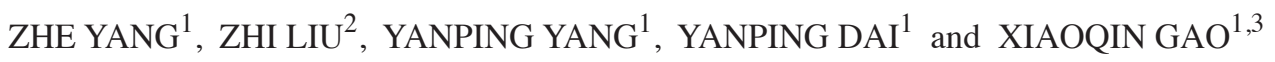 \\ ${ }^{1}$ Department of Histology and Embryology, School of Basic Medicine, Guizhou Medical University, Guiyang, \\ Guizhou 550025; ${ }^{2}$ Department of Urinary Surgery, The Second Affiliated Hospital of Guizhou Medical University, \\ Guizhou Medical University, Kaili, Guizhou 556000; ${ }^{3}$ Centre of Reproductive Research, ShenQi Ethnic Medicine College of \\ Guizhou Medical University, Guizhou Medical University, Guiyang, Guizhou 550003, P.R. China
}

Received September 23, 2020; Accepted April 20, 2021

DOI: $10.3892 / \mathrm{etm} .2021 .10279$

\begin{abstract}
Sertoli cells (SCs) are an important component of spermatogenic tubules. The blood-testis barrier (BTB) is composed of SCs and is necessary for the development and maturity of spermatogenic cells. When the tight connection between SCs is destroyed, the BTB loses its integrity, leading to impaired spermatogenesis. Polypyrimidine tract-binding protein 1 (PTBP1) is a key protein involved in precursor mRNA splicing and selective splicing events, which directly affects tumor cell proliferation and influences the formation of the blood-tumor barrier by regulating the expression levels of tight junction-associated proteins. The present study revealed that the expression of PTBP1 was downregulated following a decrease in spermatogenic activity at the phase of senescence. TM4 cells were transfected with lentivirus-short hairpinRNA-PTBP1 to evaluate the effect of silencing PTBP1 on the expression levels of tight junction proteins and the integrity of tight junctions between adjacent SCs. Western blot analysis indicated that the expression levels of Zonula occludens 1, occludin and claudin-5 decreased significantly due to silencing of PTBP1 in SCs. Through detecting trans-epithelial electrical resistance, it was revealed that silencing of PTBP1 broke the integrity of tight junctions between adjacent SCs. The results suggested that PTBP1 maintained the integrity of the BTB by promoting the expression levels of tight junction-associated proteins and revealed the possible mechanism of PTBP1 in regulating spermatogenesis.
\end{abstract}

Correspondence to: Professor Xiaoqin Gao, Department of Histology and Embryology, School of Basic Medicine, Guizhou Medical University, Dongqing Road, Guiyang, Guizhou 550025, P.R. China

E-mail: gxq550301@sina.com

Key words: polypyrimidine tract-binding protein 1, Sertoli cells, blood-testis barrier, tight junction

\section{Introduction}

Sertoli cells (SCs) are located at the base of seminiferous tubules and are important members of the wall structure. Although SCs cannot differentiate directly into sperm, they play an indispensable role in assisting and maintaining spermatogenesis (1-3). SCs perform their biological function mainly through two mechanisms, by which they secrete a variety of growth factors to establish a unique niche supporting spermatogonial stem cell self-renewal and differentiation. SCs also construct the blood-testis barrier (BTB) to separate the advanced germ cells located in the adluminal compartment from immune cells and harmful molecules in the blood, and to allow specific molecules to pass through $(1,4-7)$. The dysfunction of SCs caused by imbalances in the expression of specific genes, such as claudin-5 and JAM, is often the main factor affecting spermatogenesis $(8,9)$. Therefore, the investigation of the molecular mechanisms that influence SC function during spermiogenesis is of considerable importance.

The BTB originates from adolescence and is composed of $\mathrm{SC}$ bodies and cell junctions (including tight junctions, adherens junctions, gap junctions and desmosome-like junctions) between adjacent SCs. The tight junctions have been widely accepted as the most important structures for the BTB (10). The maintenance of the normal function of the BTB depends mainly on the expression levels of adhesion proteins and tight junction proteins, such as occludin, claudin-3, -5 and -11 , zonula occludens (ZO) 1, 2 and 3, and JAM-A and -B. Occludin and claudin-11 are responsible for the integrity of the BTB. The absence of these proteins always leads to the lack of integrity of the BTB and the increased autoimmune response of the body to spermatogenic cells, which is accompanied by decreased spermatogenesis $(1,11)$. The expression levels of these proteins are often regulated by specific transcription factors. For example, transcription factor variant 5 (ETV5) can control the expression of claudin-5. When ETV5 is knocked down, claudin-5 expression is inhibited, resulting in the destruction of the BTB (9). In addition, the transcription factor Krüppel-like factor 6, which is expressed in SCs, contributes to the maintenance of the normal function of the 
BTB by regulating the expression of the target genes ZO-1 and claudin-3 (12). It may be suggested that, to some extent, the activity of the BTB is determined by specific transcription factors.

As a heterogeneous nuclear protein, polypyrimidine tract-binding protein 1 (PTBP1) exerts its functions mainly by regulating precursor mRNA splicing and alternative splicing events $(13,14)$. PTBP1 is highly expressed in tumors of various organs, including brain $(15,16)$, colorectal $(17)$, ovarian (18), gastric (19), breast (20) and renal cancer (21). PTBP1 plays an important role in accelerating cancer cell proliferation and migration. The permeability of the blood-tumor barrier has been reported to be enhanced following knockdown of PTBP1 in endothelial glioma cells, as demonstrated in a recent study (22). This function may be closely associated with the expression of tight junction proteins, which is regulated by the transcription factor ETV1 (22). Since the function of the BTB is also highly dependent on the expression of tight junction proteins, it was hypothesized that it could be influenced by PTBP1 via regulation of the expression levels of these proteins.

In the present study, a gradual decrease in PTBP1 expression was noted, following suppression of spermatogenesis, as a result of aging. Subsequently, the expression of PTBP1 was silenced in SCs in order to investigate whether it could affect the integrity and permeability of the BTB by regulating the expression of tight junction proteins. The current study confirmed that PTBP1 served an important role in maintaining the integrity of tight junctions between adjacent SCs by promoting the expression of tight junction proteins, providing a new insight into the role of PTBP1 in regulating spermatogenesis.

\section{Materials and methods}

Isolation of primary mouse SCs. A total of $20 \mathrm{BALB} / \mathrm{c}$ mice (10 female and 10 male; 6 -week-old; $20 \mathrm{~g}$ ) were obtained from Sipeifu (Beijing) Biotechnology Co., Ltd., and were maintained in pathogen-free conditions with $50-65 \%$ humidity at $24^{\circ} \mathrm{C}$ under a 12-h light/dark cycle. The mice had free access to food and water, and were used to breed the next generation of mice. All animal experiments were performed in accordance with the guidelines of the Chinese Council on Animal Care and the present study was approved by the Guizhou Medical University Committee (permit. no. 1800013). The testes of 20 male mice (bred from the aforementioned mice; 3 -week-old; $8.5 \mathrm{~g}$ ) were collected to isolate the SCs according to a previous report $(23,24)$. Briefly, following anesthesia with sodium pentobarbital $(150 \mathrm{mg} / \mathrm{kg})$ by intraperitoneal injection, the mice were sacrificed by cervical dislocation and capsules were removed from the collected testicles. The seminiferous tubules were extracted with a $1-\mathrm{ml}$ syringe needle and subsequently cut into small fragments with ophthalmic scissors. The extract was filtered by cell filtration and digested with DMEM/Ham F-12 (DMEMF12; Gibco; Thermo Fisher Scientific, Inc.) containing collagenase IV (Gibco; Thermo Fisher Scientific, Inc.), hyaluronidase (Beijing Solarbio Science \& Technology Co., Ltd.), trypsin (Beijing Solarbio Science \& Technology Co., Ltd.) (all used at $1 \mathrm{mg} / \mathrm{ml})$ and DNase I $(0.5 \mathrm{mg} / \mathrm{ml}$; Corning, Inc.). The samples were oscillated for $20 \mathrm{~min}$ in a $37^{\circ} \mathrm{C}$ water bath. Cell pellets were collected by centrifugation $\left(500 \mathrm{x}\right.$ g for $5 \mathrm{~min}$ at $24^{\circ} \mathrm{C}$ ) and resuspended in DMEMF12 containing 15\% FBS (cat. no. 04-001-1ACS; Biological Industries) and $1 \%$ penicillin-streptomycin (Thermo Fisher Scientific, Inc.). The cells were finally cultured in a $5 \% \mathrm{CO}_{2}$ incubator at $37^{\circ} \mathrm{C}$. After culture for $48 \mathrm{~h}$, the morphology of primary SCs was observed using an inverted phase contrast microscope (magnification x100; NIKON ST100; Nikon Corporation).

Cell line culture and treatment. The mouse SC line (TM4 cells) was provided by Procell Life Science \& Technology Co., Ltd. The cells were cultured in DMEM containing 10\% FBS and $1 \%$ penicillin-streptomycin at $37^{\circ} \mathrm{C}$ in a $5 \% \mathrm{CO}_{2}$ incubator. The cells were grown to $90 \%$ confluence for subsequent experiments. To silence the expression of PTBP1, TM4 cells were infected with lentivirus (Lv)-short hairpin RNA (sh) PTBP1-EGFP (Cyagen Biosciences, Inc.) at a multiplicity of infection (MOI) of 50 at $37^{\circ} \mathrm{C}$ for $24 \mathrm{~h}$, and then cultured with fresh medium for another $48 \mathrm{~h}$ at $37^{\circ} \mathrm{C}$ in a $5 \% \mathrm{CO}_{2}$ incubator before subsequent experiments. The cells infected with Lv-EGFP (Cyagen Biosciences, Inc.; MOI, 50) were used as negative control (NC). EGFP expression was analyzed using fluorescence microscopy (magnification x100; NIKON ST100; Nikon Corporation) following transfection.

Immunostaining analysis. In a previous study (25), mice were classified into the following age groups: i) Postnatal (day 1-7); ii) Weaning (4 weeks); iii) Pubertal (6 weeks); iv) Reproductively active (15 weeks); and (v) Senescence or aged ( $>65$ weeks). Another study also reported that the rate of spermatogenesis declined at 16 months in male mice (26). Thus, in the present study, 8 -week-old mice $(n=5)$ and 18-month-old mice $(n=5)$ were purchased from Sipeifu (Beijing) Biotechnology Co., Ltd. The 8-week-old mice (between the pubertal and the reproductively active age groups) were used as the young mice, and the 18-month-old mice were used as the aged mice. Following anesthesia with sodium pentobarbital $(150 \mathrm{mg} / \mathrm{kg})$ by intraperitoneal injection, the mice were sacrificed by cervical dislocation for collection of testes. The testes collected from male mice were fixed with $4 \%$ paraformaldehyde at $24^{\circ} \mathrm{C}$ for $36 \mathrm{~h}$, dehydrated, embedded with paraffin and then sliced into $5-\mu \mathrm{m}$ tissue sections. Following incubation of the samples with $10 \%$ goat serum (cat. no. C0265; Beyotime Institute of Biotechnology) at $24^{\circ} \mathrm{C}$ for $30 \mathrm{~min}$, the sections were further incubated overnight at $4^{\circ} \mathrm{C}$ with the PTBP1 primary antibodies (1:500; cat. no. ab133734; Abcam). A second incubation was performed with FITC-tagged secondary antibodies (1:200; cat. no. A0562; Beyotime Institute of Biotechnology) at $24^{\circ} \mathrm{C}$ for $2 \mathrm{~h}$. The nuclei were stained with $300 \mathrm{nM}$ DAPI at $24^{\circ} \mathrm{C}$ for $10 \mathrm{~min}$. Similarly, the collected SCs (primary SCs) were fixed with $4 \%$ paraformaldehyde for $1 \mathrm{~h}$, incubated with $10 \%$ goat serum for $30 \mathrm{~min}$ and permeabilized with $0.15 \%$ Triton X-100 for $20 \mathrm{~min}$. These cells were incubated overnight with the primary antibodies, including PTBP1 (1:500; cat. no. ab133734; Abcam) or sex determining region Y-box 9 (SOX-9; 1:500; cat. no. ab185966; Abcam) at $4^{\circ} \mathrm{C}$ for $16 \mathrm{~h}$ and further incubated with FITC-tagged secondary antibodies (1:200; cat. no A0562; Beyotime Institute of Biotechnology) or HRP-tagged secondary antibodies (1:200; cat. no. STAR208P; Neobioscience Technology Co., Ltd.) 
at $24^{\circ} \mathrm{C}$ for $2 \mathrm{~h}$. The nuclei were stained with $300 \mathrm{nM}$ DAPI for $10 \mathrm{~min}$. The immunostaining images for SOX-9 were visualized and captured using an inverted phase contrast microscope (magnification x100; NIKON ST100; Nikon Corporation), while those for PTBP1 were visualized and captured using an inverted fluorescence microscope (magnification x100; NIKON Ti-u-dsri2; Nikon Corporation).

Western blot analysis. The total proteins were extracted from the testes and from TM4 SCs using RIPA Lysis Buffer (Beyotime Institute of Biotechnology) and the protein concentration was measured using a BCA Protein Assay kit (Beyotime Institute of Biotechnology). Following electrophoresis of the proteins (30 $\mu \mathrm{g} / \mathrm{lane})$ on $10 \%$ sodium dodecyl sulfate-polyacrylamide gels, they were transferred to PVDF membranes (EMD Millipore). The PVDF membranes were incubated with 5\% BSA (cat. no. 9048-46-8; Merck KGaA) at $24^{\circ} \mathrm{C}$ for $2 \mathrm{~h}$, primary antibodies at $4^{\circ} \mathrm{C}$ for $16 \mathrm{~h}$ and HRP-conjugated IgG antibody (1:3,500; cat. no. STAR208P; Neobioscience Technology Co., Ltd.) at $24^{\circ} \mathrm{C}$ for $2 \mathrm{~h}$. The primary antibodies used were as follows: GAPDH $(1: 1,500$; cat. no. ab181602; Abcam), PTBP1 (1:1,500; cat. no. ab133734; Abcam), ZO-1 (1:1,500; cat. no. ab216880; Abcam), occludin (1:1,500; cat. no. ab216327; Abcam) and claudin-5 (1:1,500; cat. no. ab131259; Abcam). The protein expression levels were detected using the enhanced chemiluminescence kit assay system (cat. no. CLINX-5600; Clinx Science Instruments Co., Ltd.), and the band densities were analyzed using Image Lab 4.1 (Media Cybernetics, Inc.) and standardized according to the expression levels of GAPDH.

Reverse transcription-quantitative PCR (RT-qPCR). Total RNA was extracted from testes and SCs using TRNzol Universal Total RNA extraction kit (cat. no. DP424; Tiangen Biotech Co., Ltd.) and cDNA was synthesized by FastKing-RT SuperMix (cat. no. KR118; Tiangen Biotech Co.,Ltd.) according to the manufacturer's protocol. qPCR was performed using the SuperReal PreMix Plus (SYBR Green) (cat. no. SYBR FP205; Tiangen Biotech Co., Ltd.) on ABI Prism 7900 system (Applied Biosystems; Thermo Fisher Scientific, Inc.). The qPCR thermocycling conditions were as follows: $1 \mathrm{~min}$ at $95^{\circ} \mathrm{C}$, followed by 40 cycles of $5 \mathrm{sec}$ at $95^{\circ} \mathrm{C}, 10 \mathrm{sec}$ at $55^{\circ} \mathrm{C}$ and $15 \mathrm{sec}$ at $72^{\circ} \mathrm{C}$. The aforementioned experiments were conducted according to the manufacturer's instructions. GAPDH was used as an endogenous control to normalize the gene expression data. The $2^{-\Delta \Delta \mathrm{Cq}}$ method was used to quantify the relative gene expression (27). The following primer sequences were used: GAPDH (mouse), sense (S)-5'-TGTTTCCTCGTCCCGTAG-3' and antisense (A)-5'-CAATCTCCACTTTGCCACT-3'; PTBP1 (mouse), S-5'-TCCACCCTCAGCTACCCT-3' and A-5'-CAT CTTGCGGTCCTTCTG-3'.

Cell proliferation analysis. Cell proliferation was assessed using the WST-8 Cell Counting Kit-8 (CCK-8; Dojindo Molecular Technologies, Inc.). According to the manufac-

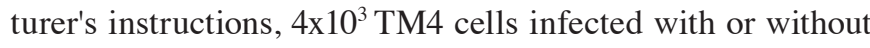
shPTBP1 were transferred in a 96-well plate and cultured for $12,24,36$ or 48 h. Subsequently, the cells were washed with PBS and incubated with $100 \mu \mathrm{l}$ serum-free medium containing $10 \mu \mathrm{l} \mathrm{CCK}-8$ solution. Following incubation at $37^{\circ} \mathrm{C}$ for $2 \mathrm{~h}$, the absorbance was measured at $450 \mathrm{~nm}$ using an Epoch 2 Microplate spectrophotometer (Biotek Epoch2).

Analysis of SC tight junction permeability in vitro. The permeability of SC tight junctions was detected using the Millicell Electrical Resistance system (MERS00002 Millicell-ERS; EMD Millipore) (12). Initially, $2.5 \times 10^{5} \mathrm{SCs}$ were plated in the Transwell upper chamber and cultured for 12, 24, 36 and $48 \mathrm{~h}$. The resistance was quantified as the trans-epithelial electrical resistance (TEER) in ohms $(\Omega)$ by placing two electrodes across the SC epithelium, one in the apical and one in the basal compartment of the bicameral units. Each sample was randomly tested three times and the average value was multiplied by the Transwell membrane area to yield the TEER value $\left(\Omega \mathrm{cm}^{2}\right)$. The final valid value was defined as the TEER value minus the TEER of the blank control.

Statistical analysis. Data were analyzed using GraphPad Prism v5.01 (GraphPad Software, Inc.) and presented as the mean \pm SEM of $\geq 3$ independent experiments. The differences between two groups were analyzed using an unpaired Student's t-test and the differences among multiple groups were determined by one-way ANOVA with Tukey's post hoc test. $\mathrm{P}<0.05$ was considered to indicate a statistically significant difference.

Laser scanning confocal microscopy and electron microcopy. The methods of laser scanning confocal microscopy and electron microcopy are described in Data S1.

\section{Results}

Expression levels of PTBP1 in mouse testicles during spermatogenesis. To determine PTBP1 expression in the testes of young and aged mice, the corresponding testicular samples were collected from 8 -week-old and 18-month-old mice. The PTBP1 expression analysis was performed using immunofluorescence assays and the BTB structures were visualized using an electron microscope. Western blotting and RT-qPCR were used to confirm the results derived from the immunofluorescence experiments. It was revealed that PTBP1 was mainly expressed in SCs of the testes, whereas the expression levels of PTBP1 were gradually decreased when the spermatogenic ability was decreased following aging of the mice (Fig. 1A-C). In addition, the formation of BTB structures was impaired in the testes from 18-month-old mice (Fig. S1). Furthermore, primary mouse SCs isolated from 3-week-old mice were successfully isolated and cultured; these cells were characterized by expression of the SC-specific molecule SOX-9 and Wilms tumor 1 (Wt1) (Figs. 2A and B, and S2). The expression of PTBP1 was confirmed in the nucleus of mouse primary SCs and TM4 cells (Fig. 2C). The aforementioned results demonstrated that SCs were the main cells expressing PTBP1 in the testes. Due to the important role of SCs in spermatogenesis, the present study further investigated whether PTBP1 was involved in regulating spermatogenesis by affecting the biological function of SCs.

PTBP1 regulates the proliferation of SCs. In previous studies, PTBP1 was shown to promote the proliferation of various cell types $(16,18)$. SC proliferation is an important prerequisite 
A

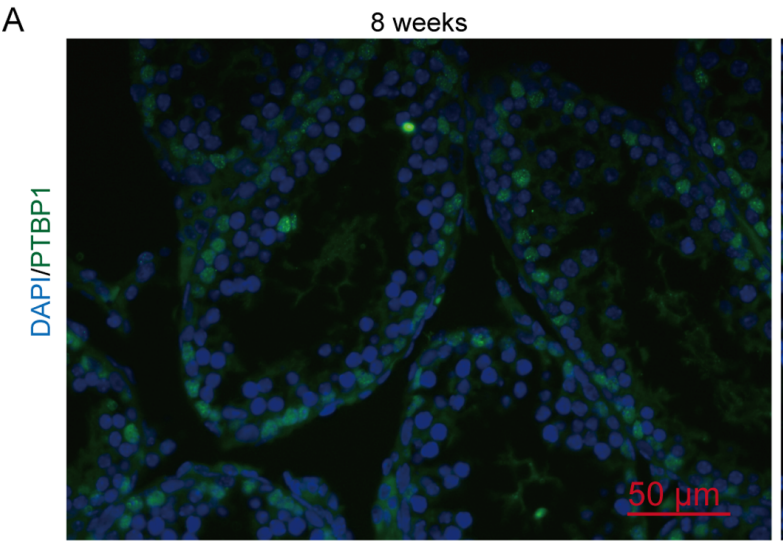

B

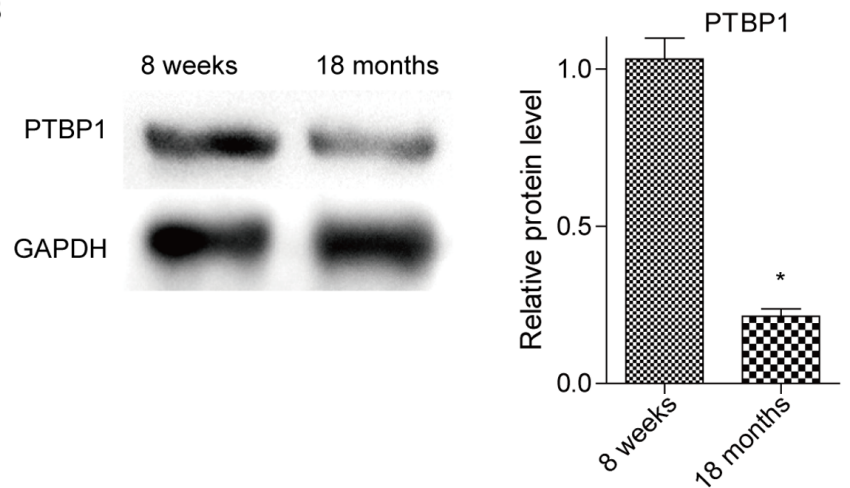

18 months

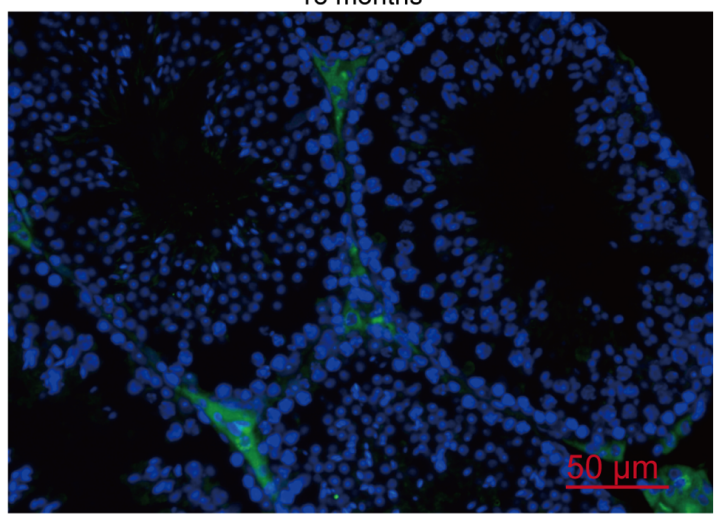

C

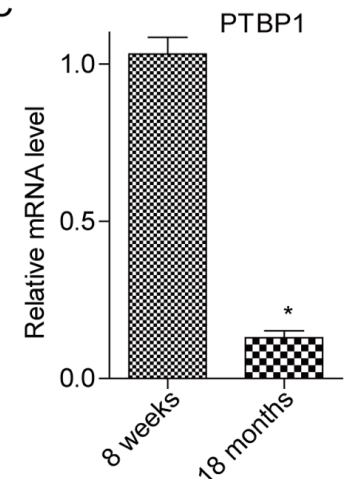

Figure 1. Expression levels of PTBP1 in the testes of adult and aged mice. (A) Protein expression levels of PTBP1 in the testes were detected by immunofluorescence assays at 8 weeks and 18 months after birth. (B) PTBP1 expression in the testicular tissues was detected by western blot analysis at 8 weeks and 18 months after birth. (C) PTBP1 expression in the testicular tissues was detected by reverse transcription-quantitative PCR at 8 weeks and 18 months after birth. Scale bar, $50 \mu \mathrm{m}$. Data are presented as the mean \pm SEM $(n=5)$. ${ }^{*} \mathrm{P}<0.05$ vs. 8 weeks. PTBP1, polypyrimidine tract-binding protein 1 .

for ensuring appropriate spermatogenesis; therefore, the present study investigated the role of PTBP1 in regulating $\mathrm{SC}$ proliferation. In the present study, the expression levels of PTBP1 were inhibited by infecting TM4 cells with Lv-shPTBP1-EGFP; infection of cells with Lv-EGFP was used as NC. Following transfection for 3 days, the expression levels of EGFP were assessed using fluorescence microscopy (Fig. 3A). Concomitantly, the expression levels of PTBP1 were knocked down (Fig. 3B). Cell proliferation was assessed with the CCK-8 assay and the results indicated that suppression of PTBP1 expression resulted in decreased cell proliferation (Fig. 3C). These findings suggested that PTBP1 may serve an important role in regulating SC proliferation.

PTBPl is necessary to maintain the integrity and regulate the permeability of the $B T B$. The integrity and permeability of the BTB are very important to maintain the appropriate environment required for spermatogenesis $(4,6,8)$. Therefore, the present study explored the regulatory effect of PTBP1 on the integrity and permeability of the BTB using an in vitro cell model. Following infection of TM4 cells with Lv-shPTBP1-EGFP or Lv-EGFP, changes in the integrity and permeability of the tight connections between adjacent SCs were detected using the Millicell Electrical Resistance system. The results indicated that the resistance across the epithelium was significantly decreased following the inhibition of PTBP1 expression (Fig. 4A). These results indicated that PTBP1 could maintain the tight junctions of SCs, and may have an important role in preserving the integrity of the BTB and inhibiting its permeability.

$P T B P 1$ regulates the expression of $B T B$-associated proteins in SCs. SCs are the main structural components of the BTB, whose function mainly depends on the expression of various BTB-associated proteins, including adhesive proteins and tight connection molecules (ZO-1, occludin and claudin-5) (9-11). In order to assess the effects of PTBP1 on the expression of BTB-associated molecules, TM4 cells were infected with Lv-shPTBP1-EGFP or Lv-shPTBP1-EGFP and cultured for 3 days. The proteins were extracted to assess the expression levels of ZO-1, occludin and claudin-5 by western blot analysis. As shown in Fig. 4B, PTBP1 silencing resulted in a significant decrease in the protein expression levels of ZO-1, occludin and claudin-5. These results suggested that PTBP1 may maintain the integrity and permeability of the BTB by controlling the expression of tight connection-associated molecules.

\section{Discussion}

Spermatogonial stem cells (SSCs) in spermatogenic tubules undergo proliferation, differentiation and morphological changes to form sperm. During spermatogenesis, a series of changes in SSCs are mainly dependent on the tissue microenvironment, which is predominantly composed 
A

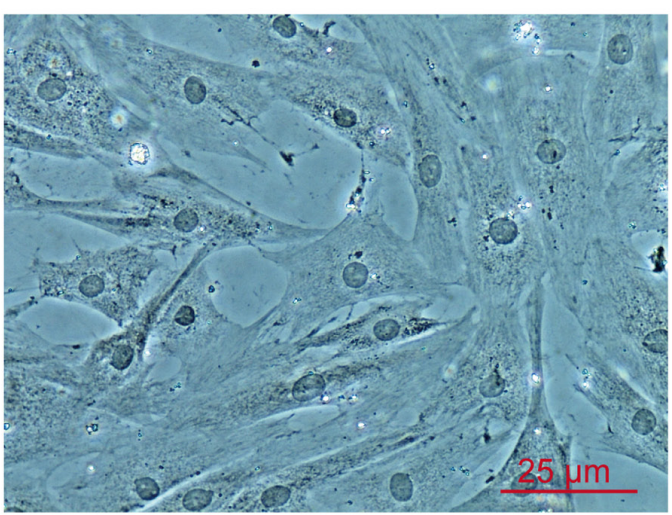

B

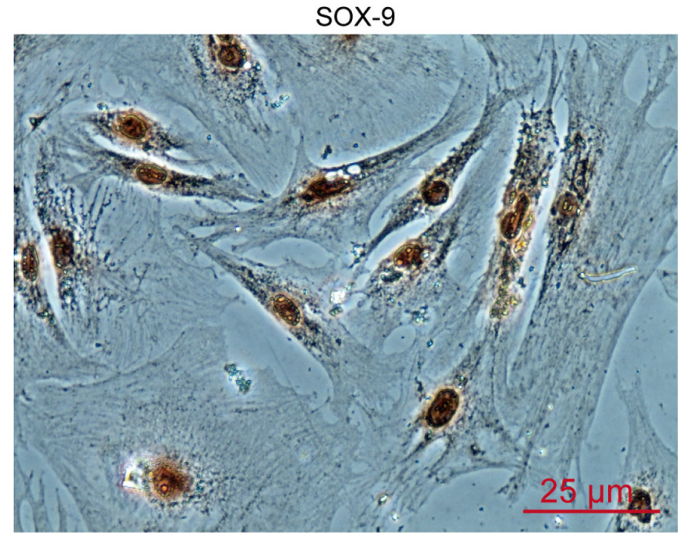

C
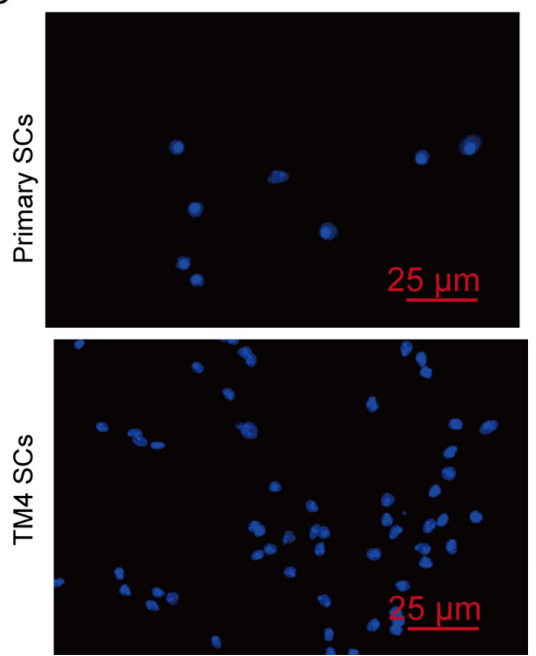
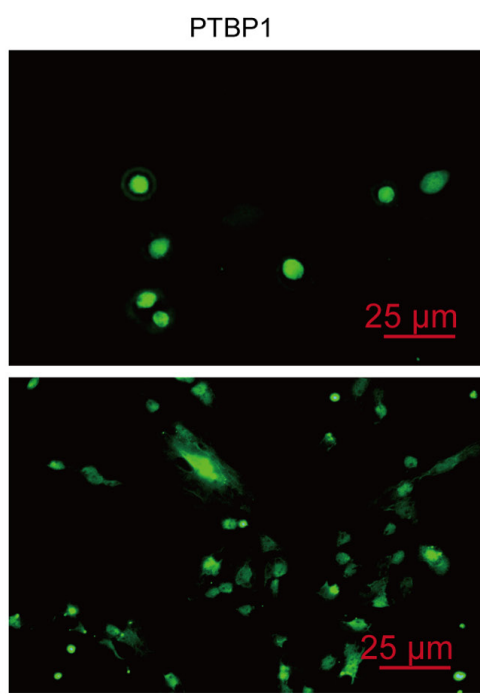
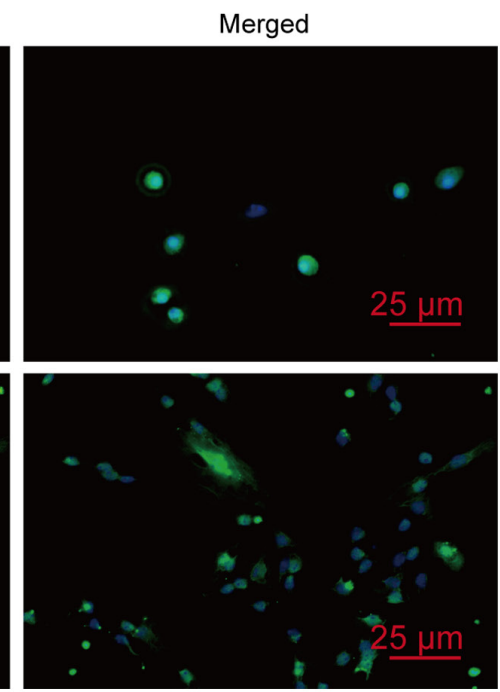

Figure 2. Expression of PTBP1 in SCs. (A) Morphology of primary SCs was observed using an optical microscope following isolation and culture for 48 h. (B) Immunocytochemistry was used to evaluate the expression levels of the cell-specific molecule SOX-9 in primary mouse SCs. (C) Immunofluorescence was used to assess the expression of PTBP1 in primary SCs and TM4 SCs. Scale bar, $25 \mu \mathrm{m}$. PTBP1, polypyrimidine tract-binding protein 1; SCs, Sertoli cells; SOX-9, sex determining region Y-box 9.

of SC-secreted growth factors, basal membrane and the components exuded from blood vessels $(1,28-31)$. Due to the specific histological association between SCs and SSCs, the former type of cells may be the most important contributor to the tissue microenvironment of spermatogenic cells. SCs not only provide autocrine/paracrine signals, but also secrete basement membrane components. Moreover, adjacent SCs form the physical barrier, the BTB, which inhibits autoimmune responses to the germ cells by the formation of tight junctions (1). Spermatogenesis is accompanied by dynamic changes in the wall structure of spermatogenic tubules and notably in the relevant molecules of the BTB that support spermatogenesis. These changes are closely associated with the spatial and temporal expression of various genes (10). Therefore, it is of considerable importance to investigate the molecular mechanism that regulates the BTB in order to fully understand the regulatory mechanism of spermatogenesis.

PTBP1 is an RNA-binding protein that exerts its function by affecting mRNA splicing stability and translation (13). PTBP1 regulates a series of biological processes, including maintenance of cellular structure and movement, immunity, protein metabolism and the cell cycle $(14,32,33)$. Previous studies examined the ability of PTBP1 to regulate cancer development and immune system function, and revealed that it could directly accelerate proliferation and metastasis of tumor cells, and promote the development of multiple tumors and the enhancement of drug resistance by affecting the tumor inflammatory microenvironment and the permeability of the blood-tumor barrier (22). In addition, the mechanism of action of PTBP1 was assessed with regard to glioma formation and the results indicated that increased expression of PTBP1 inhibited the permeability of the blood-tumor barrier of glioma, leading to the decreased effectiveness of anti-tumor drug therapy. These effects were closely associated with the expression of the tight junction protein regulated by the circular RNA_001160/microRNA-195-5p/ETV1 signaling cascade, which was in turn mediated by PTBP1 (22). However, whether PTBP1 is involved in the regulation of the BTB for maintaining spermatogenesis remains unclear.

In the present study, the data indicated that the expression of PTBP1 was decreased with the reduction in spermatogenic activity following aging. The results further confirmed that PTBP1 was expressed in the nuclei of SCs. Testicular maturation is accompanied by the proliferation of SCs and by formation of the BTB. In response to aging, the degeneration of SC function and the loss of BTB integrity may be important 
A
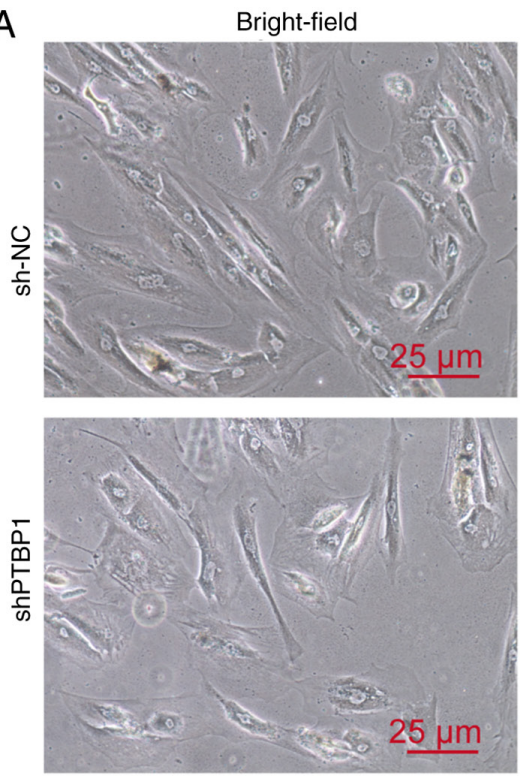
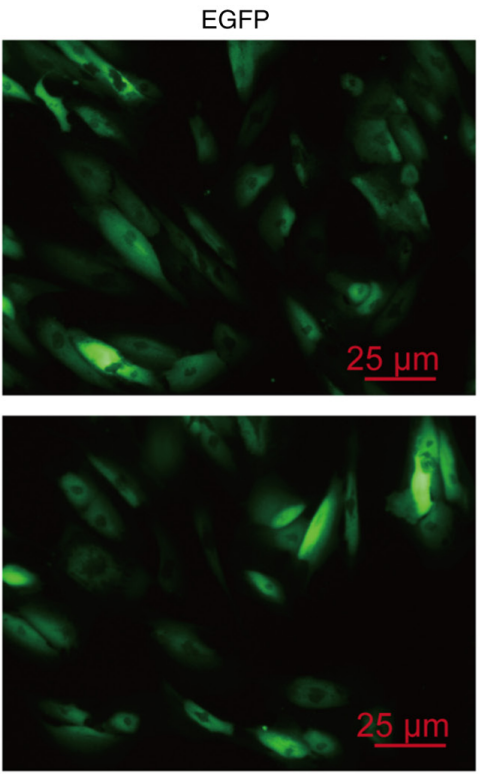
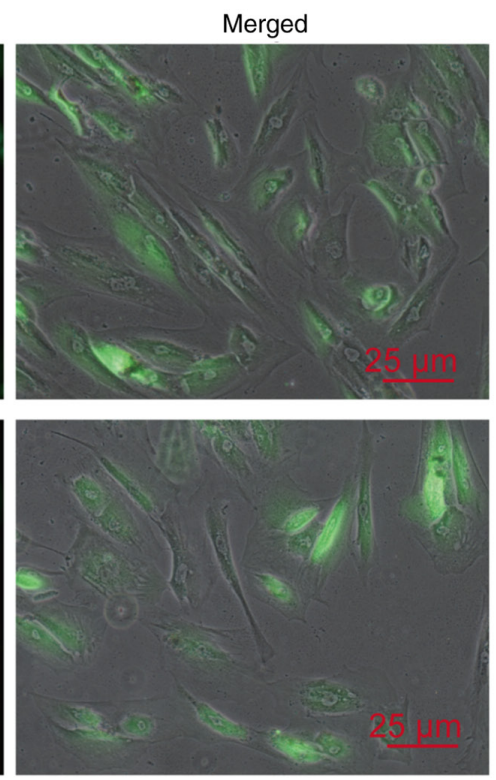
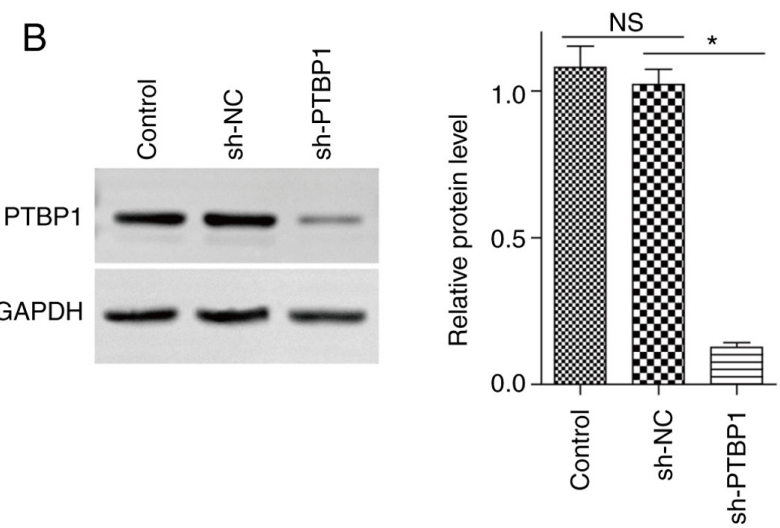

C

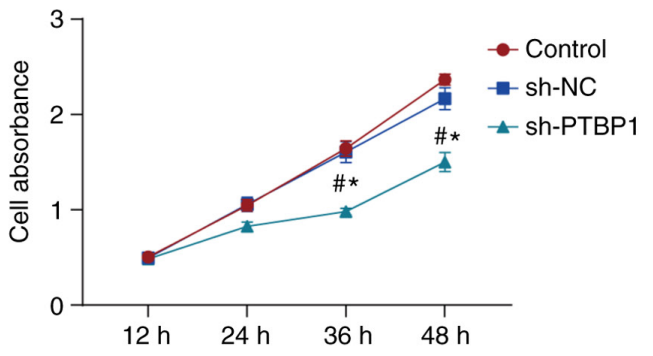

Figure 3. Effects of PTBP1 on the proliferation of SCs. The expression levels of PTBP1 were knocked down in TM4 SCs by infection of the cells with Lv-sh PTBP1-EGFP (sh-PTBP1). Cells infected with Lv-EGFP (sh-NC) were used as NC cells. (A) After infection, the cells were incubated for 3 days and the expression levels of EGFP were observed under a fluorescence microscope. (B) After infection with Lv-sh PTBP1-EGFP or Lv-EGFP, the cells were cultured for 3 days and PTBP1 expression was observed by western blotting. (C) Stable PTBP1-knockdown TM4 SCs or the corresponding control cells were seeded in a 96-well plate and cell viability was detected following culture for 12, 24, 36 and $48 \mathrm{~h}$. Scale bar, $25 \mu \mathrm{m}$. Data are presented as the mean $\pm \mathrm{SEM}(\mathrm{n}=3)$. ${ }^{\prime} \mathrm{P}<0.05$ vs. the blank group (control); " $\mathrm{P}<0.05$ vs. the sh-NC group. PTBP1, polypyrimidine tract-binding protein 1; SCs, Sertoli cells; NC, negative control; NS, not significant; sh, short hairpin RNA; Lv, lentivirus.
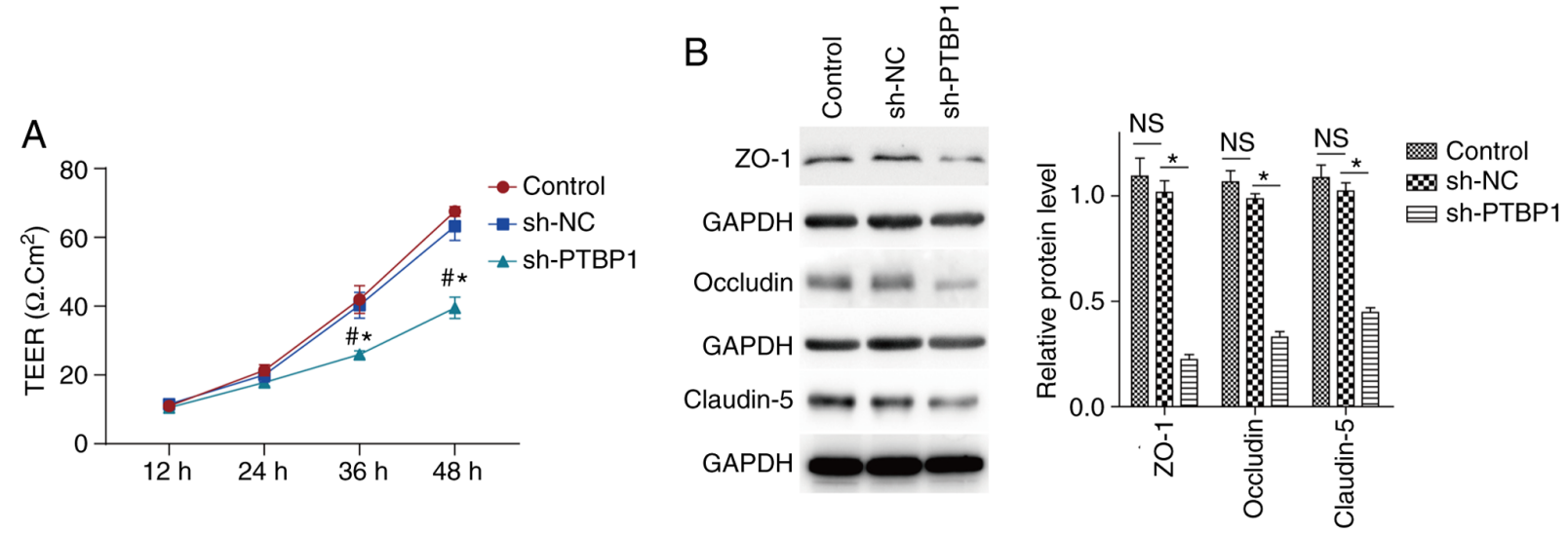

Figure 4. Knockdown of PTBP1 increases BTB permeability in vitro and decreases the expression levels of tight junction-associated proteins. (A) Following stable PTBP1-knockdown in TM4 SCs, the infected cells and their corresponding controls were seeded in a 96-well plate for 12, 24, 36 and 48 h. The permeability of the tight junction was measured by a Millicell Electrical Resistance system. Non-infected TM4 SCs were used as a blank control. (B) Protein expression levels of ZO-1, occludin and claudin-5 were detected by western blot analysis following infection of TM4 SCs with Lv-sh PTBP1-EGFP or Lv-EGFP for 3 days. Non-infected TM4 SCs were used as a blank control. Data are presented as the mean $\pm \mathrm{SEM}(\mathrm{n}=3)$. ${ }^{*} \mathrm{P}<0.05$ vs. the blank group (control); ${ }^{*} \mathrm{P}<0.05$ vs. the sh-NC group. PTBP1, polypyrimidine tract-binding protein 1; BTB, blood-testicular barrier; SCs, Sertoli cells; NC, negative control; NS, not significant; sh, short hairpin RNA; TEER, trans-epithelial electrical resistance; ZO-1, zona occludens 1. 
factors leading to the weakening of spermatogenesis. Based on this evidence, the present study hypothesized that the dynamic changes in PTBP1 expression may be an important regulatory factor affecting the biological function of SCs. To assess this hypothesis, gene editing was used to silence the expression of the PTBP1 gene in TM4 cells and to further detect the changes in cell viability and integrity of the BTB. The results indicated that PTBP1 played an important role in maintaining the proliferation of SCs, whereas PTBP1 silencing caused a significant increase in the permeability of the BTB. In addition, the effects of PTBP1 silencing were investigated on the expression of BTB-associated proteins in SCs. PTBP1 silencing resulted in decreased expression levels of ZO-1, occludin and claudin-5 in SCs. These results further supported the conclusion that PTBP1 may maintain the integrity of the BTB by regulating the expression of ZO-1, occludin and claudin-5.

Despite the significant findings, several issues remain unresolved. Firstly, the signaling mechanisms responsible for inducing the dynamic changes in PTBP1 expression during spermatogenesis were not fully clarified. It is suspected that the changes in the proportion and function of spermatogenic cells in the testicular tissues during the flourishing and declining stages of spermatogenesis may be the cause of the dynamic changes in PTBP1 expression. Secondly, the exact mechanism by which PTBP1 affects the expression of BTB-associated proteins was not fully clarified. A previous study (22) has also demonstrated the direct or indirect regulatory effect of PTBP1 on the expression of specific genes. For example, in glioma vascular endothelial cells, PTBP1 and the transcription factor ETV5 were revealed to cooperate to regulate the expression of tight junction proteins (ZO-1, occludin, and claudin-5) (22). Therefore, it is possible that such biological effects may also exist in SCs, but the association between PTBP1 and ETV1 requires further elucidation. Whether pharmacological intervention of PTBP1 activity can restore the dysfunction of SCs to ultimately improve spermiogenesis remains unknown; these topics will be further addressed in future studies.

\section{Acknowledgements}

The authors would like to thank the Research Center for Basic Sciences of Medicine of Guizhou Medical University for providing experimental equipment, such as the fluorescence microscope (NIKON Ti-u-dsri2; Nikon Corporation) and the laser scanning confocal fluorescence microscope (Olympus FV1000; Olympus Corporation).

\section{Funding}

The present study was financially supported by grants from the Guizhou Provincial Education Department Youth Science and Technology talent Growth Project [grant no. KY (2018)172] and the Science and Technology Joint Fund Project in Guizhou Province [grant. no. LH (2016)7386].

\section{Availability of data and materials}

The datasets used and/or analyzed during the current study are available from the corresponding author on reasonable request.

\section{Authors' contributions}

$Z Y$ and $X G$ conceived and designed the experiments. ZY, ZL, YY and YD performed the experiments. ZY and ZL analyzed the data. ZY and XG prepared the manuscript. All contributing authors have participated in the study, and concur with the submission and subsequent revisions submitted. XG and ZY confirm the authenticity of all the raw data. All authors read and approved the final manuscript.

\section{Ethics approval and consent to participate}

All animal experiments were performed in accordance with the guidelines of the Chinese Council on Animal Care and the present study was approved by the Guizhou Medical University Committee (permit. no. 1800013).

\section{Patient consent for publication}

Not applicable.

\section{Competing interests}

The authors declare that they have no competing interests.

\section{References}

1. França LR, Hess RA, Dufour JM, Hofmann MC and Griswold MD: The sertoli cell: One hundred fifty years of beauty and plasticity. Andrology 4: 189-212, 2016.

2. Meroni SB, Galardo MN, Rindone G, Gorga A, Riera MF and Cigorraga SB: Molecular mechanisms and signaling pathways involved in sertoli cell proliferation. Front Endocrinol (Lausanne) 10: 224, 2019.

3. Griswold MD: 50 years of spermatogenesis: Sertoli cells and their interactions with germ cells. Biol Reprod 99: 87-100, 2018.

4. Dym M and Fawcett DW: The blood-testis barrier in the rat and the physiological compartmentation of the seminiferous epithelium. Biol Reprod 3: 308-326, 1970.

5. Russell LD and Clermont Y: Degeneration of germ cells in normal, hypophysectomized and hormone treated hypophysectomized rats. Anat Rec 187: 347-366, 1977.

6. Mruk DD and Cheng CY: Tight junctions in the testis: New perspectives. Philos Trans R Soc Lond B Biol Sci 365: 1621-1635, 2010.

7. Doyle TJ, Kaur G, Putrevu SM, Dyson EL, Dyson M, McCunniff WT, Pasham MR, Kim KH and Dufour JM: Immunoprotective properties of primary Sertoli cells in mice: Potential functional pathways that confer immune privilege. Biol Reprod 86: 1-14, 2012.

8. Shao M, Ghosh A, Cooke VG, Naik UP and Martin-DeLeon PA: JAM-A is present in mammalian spermatozoa where it is essential for normal motility. Dev Biol 313: 246-255, 2008.

9. Morrow CM, Tyagi G, Simon L, Carnes K, Murphy,KM Cooke PS, Hofmann MCC and Hess RA: Claudin 5 expression in mouse seminiferous epithelium is dependent upon the transcription factor ets variant 5 and contributes to blood-testis barrier function. Biol Reprod 81: 871-879, 2009.

10. Mital P, Hinton BT and Dufour JM: The blood-testis and blood-epididymis barriers are more than just their tight junctions. Biol Reprod 84: 851-858, 2011.

11. Gow A, Southwood CM, Li JS, Pariali M, Riordan GP Brodie SE, Danias J, Bronstein JM, Kachar B and Lazzarini RA: CNS myelin and sertoli cell tight junction strands are absent in Osp/claudin-11 null mice. Cell 99: 649-659, 1999.

12. Wang XX, Zhang Y, Li XY, Li J, Tang JX, Li YY, Deng SL, Cheng CY and Liu YX: Kruppel-like factor 6 regulates Sertoli cell blood-testis barrier. Front Biosci (Landmark Ed) 24: 1316-1329, 2019.

13. Zhang L, Yang Z, Huang W and Wu J: H19 potentiates let-7 family expression through reducing PTBP1 binding to their precursors in cholestasis. Cell Death Dis 10: 168, 2019. 
14. Glisovic T, Bachorik JL, Yong J and Dreyfuss G: RNA-binding proteins and post-transcriptional gene regulation. FEBS Lett 582: 1977-1986, 2008.

15. McCutcheon IE, Hentschel SJ, Fuller GN, Jin W and Cote GJ: Expression of the splicing regulator polypyrimidine tract-binding protein in normal and neoplastic brain. Neuro Oncol 6: 9-14, 2004.

16. Cheung HC, Hai T, Zhu W, Baggerly KA, Tsavachidis S, Krahe R and Cote GJ: Splicing factors PTBP1 and PTBP2 promote proliferation and migration of glioma cell lines. Brain 132: 2277-2288, 2009.

17. Takahashi H, Nishimura J, Kagawa Y, Kano Y, Takahashi Y, Wu X, Hiraki M, Hamabe A, Konno M, Haraguchi N, et al: Significance of polypyrimidine tract-binding protein 1 expression in colorectal cancer. Mol Cancer Ther 14: 1705-1716, 2015.

18. He X, Pool M, Darcy KM, Lim SB, Auersperg N, Coon JS and Beck WT: Knockdown of polypyrimidine tract-binding protein suppresses ovarian tumor cell growth and invasiveness in vitro. Oncogene 26: 4961-4968, 2007.

19. Sugiyama T, Taniguchi K, Matsuhashi N, Tajirika T, Futamura M, Takai T, Akao Y and Yoshida K: miR-133b inhibits growth of human gastric cancer cells by silencing pyruvate kinase muscle-splicer polypyrimidine tract-binding protein 1 . Cancer Sci 107: 1767-1775, 2016

20. He X, Arslan AD, Ho TT, Yuan C, Stampfer MR and Beck WT: Involvement of polypyrimidine tract-binding protein (PTBP1) in maintaining breast cancer cell growth and malignant properties. Oncogenesis 3: e84, 2014.

21. Shan H, Hou P, Zhang M, Li L, Pan Y, Chen F and Jiang T: PTBP1 knockdown in renal cell carcinoma inhibits cell migration, invasion and angiogenesis in vitro and metastasis in vivo via the hypoxia inducible factor-1 $\alpha$ pathway. Int J Oncol 52: 1613-1622, 2018.

22. Li H, Shen S, Ruan X, Liu X, Zheng J, Liu Y, Yang C, Wang D, Liu L, Ma J, et al: Biosynthetic CircRNA 001160 induced by PTBP1 regulates the permeability of BTB via the CircRNA_001160/miR-195-5p/ETV1 axis. Cell Death Dis 10 $960,2019$.
23. Cai H, Ren Y, Li XX, Yang JL, Zhang CP, Chen M, Fan $\mathrm{CH}$, Hu XQ, Hu ZY, Gao F and Liu YX: Scrotal heat stress causes a transient alteration in tight junctions and induction of TGF- $\beta$ expression. Int J Androl 34: 352-362, 2011.

24. Li XX, Chen SR, Shen B, Yang JL, Ji SY, Wen Q, Zheng QS, Li L, Zhang J, Hu ZY, et al: The heat-induced reversible change in the blood-testis barrier (BTB) is regulated by the androgen receptor (AR) via the partitioning-defective protein (Par) polarity complex in the mouse. Biol Reprod 89: 12, 2013.

25. Choubey M, Ranjan A, Bora PS, Baltazar F, Martin LJ and Krishna A: Role of adiponectin as a modulator of testicular function during aging in mice. Biochim Biophys Acta Mol Basis Dis 1865: 413-427, 2019.

26. Hacker-Klom UB: Age dependence of murine spermatogenesis. Z Naturforsch C J Biosci 50: 303-310, 1995.

27. Livak KJ and Schmittgen TD: Analysis of relative gene expression data using real-time quantitative PCR and the 2(-Delta Delta C(T)) method. Methods 25: 402-408, 2001

28. Chiarini-Garcia H, Hornick JR, Griswold MD and Russell LD: Distribution of type A spermatogonia in the mouse is not random. Biol Reprod 65: 1179-1185, 2001.

29. Chiarini-Garcia H, Raymer AM and Russell LD: Non-random distribution of spermatogonia in rats: Evidence of niches in the seminiferous tubules. Reproduction 126: 669-680, 2003.

30. Kanatsu-Shinohara M, Inoue K, Ogonuki N, Miki H, Yoshida S, Toyokuni S, Lee J, Ogura A and Shinohara T: Leukemia inhibitory factor enhances formation of germ cell colonies in neonatal mouse testis culture. Biol Reprod 76: 55-62, 2007.

31. Oatley JM and Brinster RL: The germline stem cell niche unit in mammalian testes. Physiol Rev 92: 577-595, 2012.

32. Georgilis A, Klotz S, Hanley CJ, Herranz N, Weirich B, Morancho B, Leote AC, D'Artista L, Gallage S and Seehawer M: PTBP1-mediated alternative splicing regulates the inflammatory secretome and the pro-tumorigenic effects of senescent cells Cancer Cell 34: 85-102, 2018.

33. Wu P, Gao Y, Shen S, Xue Y, Liu X, Ruan X, Shao L, Liu Y and Wang P: KHDRBS3 regulates the permeability of blood-tumor barrier via cDENND4C/miR-577 axis. Cell Death Dis 10: 536, 2019. 